Nephrologe 2011 · 6:107-107 DOI 10.1007/s11560-010-0502-6

Online publiziert: 26. Januar 2011

(c) Springer-Verlag 2011

\author{
D. Fliser \\ Klinik für Innere Medizin IV, Universitätsklinikum des Saarlandes, Homburg/Saar
}

\title{
Intensivmedizin in der Nephrologie
}

Kaum ein anderes Gebiet der Nephrologie hat in den letzten Jahren so viel Umbruch erlebt wie das akute Nierenversagen und die extrakorporale Therapie auf der Intensivstation. Neue Definitionen des akuten Nierenversagens (RIFLE und AKIN) führen weltweit zur Standardisierung von Studienprotokollen und zum besseren Vergleich von Studienergebnissen. Neue Biomarker des akuten Nierenversagens (NGAL) werden in ihrer Voraussagekraft für die Entwicklung eines akuten Nierenversagens und für die Prognose der $\mathrm{Pa}$ tienten in prospektiven Studien getestet. Neue Verfahren der Nierenersatztherapie (SLED) haben sich zwischenzeitlich etabliert und werden routinemäßig auf der Intensivstation angewendet. Neuere Verfahren der Antikoagulation (Zitrat) werden jetzt auch auf ihre Tauglichkeit für die extrakorporale Therapie in der Intensivmedizin getestet. Nicht zuletzt wurde mittlerweile auch die Frage, welche (Mindest-)Dosis der Nierenersatztherapie bei kritisch Kranken notwendig ist, in großen prospektiven randomisierten Studien hinreichend geklärt.

Diese und viele andere wichtige Aspekte der nephrologischen Intensivmedizin wie z. B. die Prophylaxe des akuten Nierenversagens werden in den Beiträgen dieses Schwerpunkthefts erörtert. Abgerundet wird das Leitthema durch einen Überblick über die extrakorporale Therapie von Autoimmunerkrankungen mittels Plasmapherese und Immunadsorption, die für uns Nephrologen zunehmend zum wichtigen Standbein in der Intensivmedizin werden. Angesichts dieser Fülle von neuen Möglichkeiten in der nephrologischen Diagnostik und Therapie von kritisch kranken Patienten scheint die Zeit gereift zu sein, dass die Nephrologie in der Intensivmedizin nicht weiter Terrain an andere Disziplinen verliert.

Ich wünsche Ihnen auch diesmal viel Vergnügen beim Lesen!

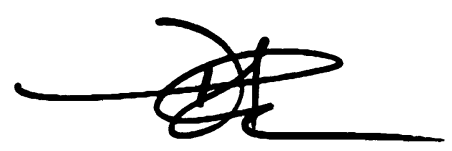

Prof. Dr. Danilo Fliser, Homburg/Saar

\section{Korrespondenzadresse \\ Prof. Dr. D. Fliser}

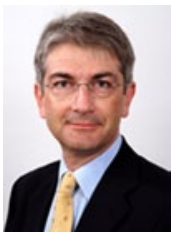

Klinik für Innere Medizin IV, Universitätsklinikum des Saarlandes Kirrberger Straße, 66421 Homburg/Saar Danilo.Fliser@uniklinikumsaarland.de 